\title{
Retreatment of permanent incisors with fibre and cast metalposts
}

\author{
Karan Bansal $^{1^{*}}$, B P Bansal ${ }^{2}$, Neha Aggarwal $^{3}$, Muskaan Nischal ${ }^{4}$ \\ ${ }^{1}$ Senior Resident, ${ }^{2,3}$ Private Practitioner, ${ }^{4}$ Post Graduate Student, Dept. of Conservative Dentistry and Endodontics, ${ }^{1}$ Adesh Institute of \\ Dental Sciences and Research, Bathinda, Punjab, ${ }^{4}$ Sri Guru Ram Das Institute of Medical Sciences \& Research, Amritsar, Punjab, India
}

\section{*Corresponding Author: Karan Bansal}

Email: drkaranbansal@ymail.com

\begin{abstract}
This case report describes the management of a supragingivally complicated crown fracture of a maxillary central incisors. The patient aged 12 years gave a history of trauma one year back and got the root canal treatment done. The patient gave a history of not getting the treatment completed after the obturations were done and that led to furthur loss of unsupported tooth structure. In this case fibre post was used in 11 . The tooth structure of 21 could not be supported by fiber post so crown lengthening followed by cast metal post and core was given. The teeth were further restored with porcelain fused metal crowns.
\end{abstract}

Keywords: Crown fracture, Dental trauma, Fiber reinforced post, Cast metal post, Crown lengthening.

\section{Introduction}

A good aesthetic restoration includes the gingival unit, the restored tooth and its interface. In a tooth with fracture below the alveolar bone crest, Orthodontic eruption can be a useful approach without compromising on the aesthetics. It is an renowned clinical treatment module for altering the relation between a non-restorable tooth and its attachment apparatus, elevating the sound tooth structure from the alveolar socket, which creates a ferrule effect that is vital for the optimal biomechanical performance of the restored teeth. ${ }^{1}$

This case report presents a case of retreatment of fractured 11 and 21 . The patient aged 12 years gave a history of trauma one year back. The root canal treatment was done. The patient gave a history of incomplete treatment after the obturations were done and that led to further loss of unsupported tooth structure.

\section{Discussion}

The purpose of a post is to retain a core in a tooth with extensive loss of coronal structure. However, while preparing the post-space, procedural accidents may occur which poses an additional risk to a restorative procedure. Rarely, these mishaps lead to a perforation in the apical portion of the root so called as "strip perforation." The post sometimes can increase the chances of root fracture and treatment failure particularly if an oversized post channel is prepared. $^{2}$

Thus, posts should only be used when other options are not available in order to retain a core. In this case, a fibre post was placed in maxillary right central incisor. The tooth structure of maxillary left central incisor could not be supported by fiber post so crown lengthening followed by cast metal post and core was the treatment approach followed.

A post is of little significance in a structurally sound anterior tooth and it also further increases the chances of a nonrestorable failure. The same holds true for an anterior tooth with a porcelain veneer. ${ }^{3}$
For restoring an endodontically treated anterior tooth with a crown, often a post is indicated. Generally after rootcanal treatment, the residual coronal tooth structure is reduced and thus to restore it to its optimal functional and structural form, a post is placed followed by a crown. The residual coronal tooth structure and the functional requirements of the tooth ascertain to whether an anterior tooth requires a post. ${ }^{4}$ As anterior teeth have to resist both lateral and shearing forces, and also the pulp chambers are very small to provide adequate resistance and retention, a post is often recommended.

Many studies have reported a high success rate with cast post and cores. They provide advantages in certain clinical scenarios. For example, when many teeth require posts, it is sometimes more sensible to make an impression and fabricate in the laboratory rather than doing a chair-side procedure. ${ }^{5}$ [Weine FS, 1995; Drummond JL 2000]. A cast post and core is also indicated when a tooth is not properly aligned and then the core has to be angled in relation to the post to acquire appropriate alignment with the adjacent teeth. Cast post and cores are also indicated in smaller teeth such as mandibular incisors, where there is a minimal coronal tooth structure available to resist rotational forces. Cast post and cores are easy to retrieve in case an endodontic retreatment is required. ${ }^{6}$

Carbon fiber posts which became popular were more flexible than metal posts and had almost the same modulus of elasticity (stiffness) as that of dentin. When bonded with resin cement, the forces are more evenly distributed in the root, causing lesser root fractures. 7

Re treatment of permanent incisors follow by cast metal post and core 


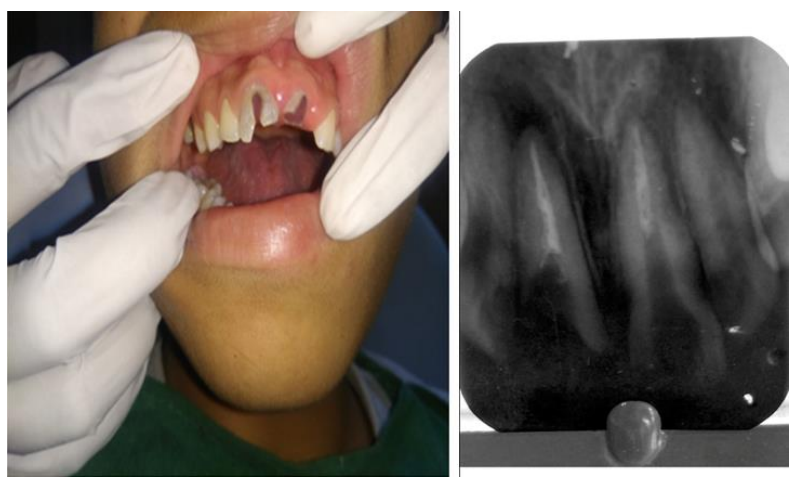

Fig. 1: (a) \& (b) Pre op photograph and iopa

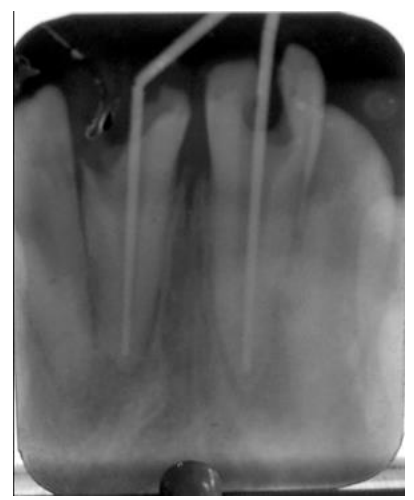

(c) Removal of GP points followed by iopa with master cones.

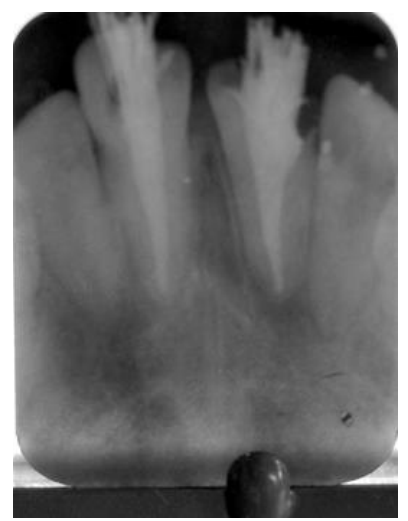

(d) Obturating 11 and 21

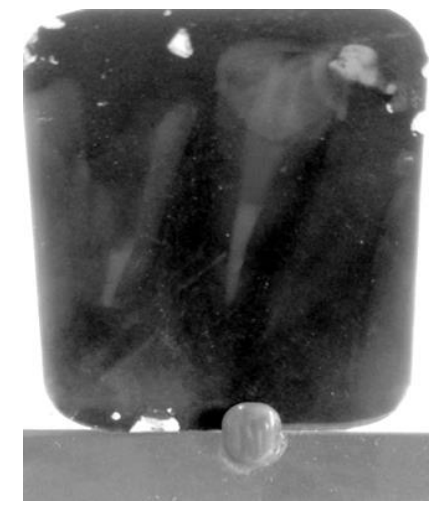

(e) Fibre post placed in 11 and post space prepared for metal post in 21

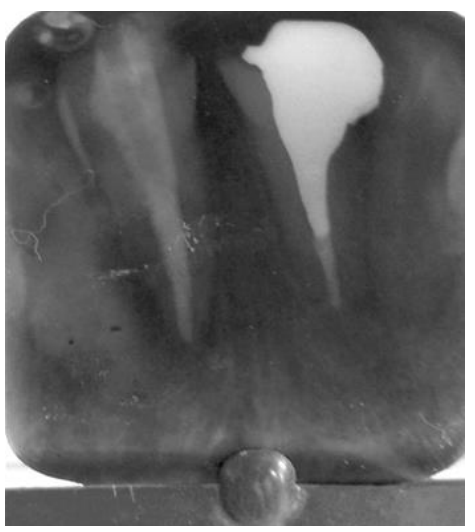

(f) Cast metal post luted in 21

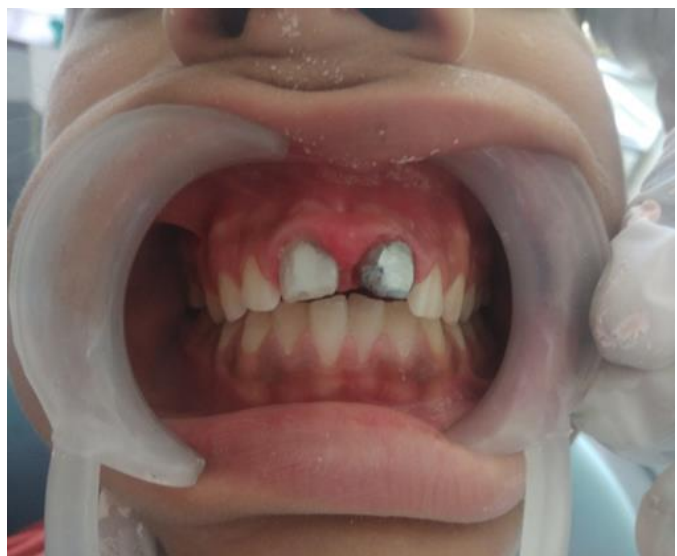

(h) Crown cuttings done after fiber post luting w.r.t 11 and cast metal post w.r.t 21

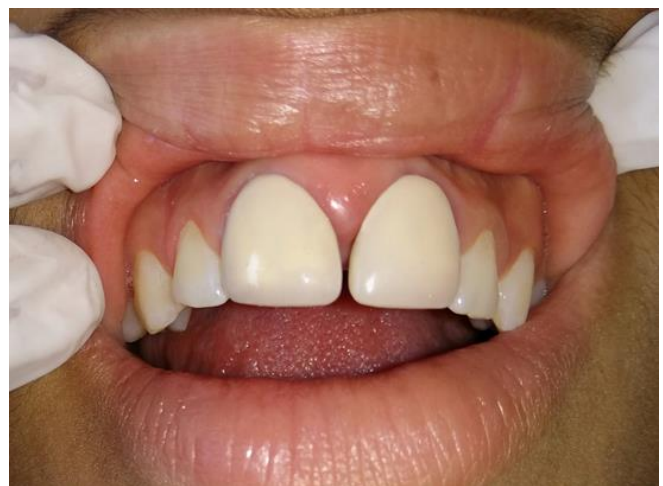

(i) Post op phoographh with PFM crowns w.r.t 11 and 21.

\section{Conclusion}

It is possible to achieve high levels of clinical success with most of the current restorative systems if certain basic rationale is followed in the restoration of endodontically treated teeth. Clinically fiber posts are routinely used. Their performance in vitro is comparable to that of metal posts and studies agree that their failure mode is more favorable than with metal posts.

The use of fiber posts will probably continue to grow, assuming that future long-term clinical research studies report similar success levels as seen in the relatively short- 
term studies already published. Further modifications of their physical and mechanical properties will probably also improve their clinical outcome.

\section{Source of funding}

None.

\section{Conflict of interest}

None.

\section{References}

1. Burke FJ. Tooth fracture In vivo and in vitro. J Dent 1992;20:131-9.

2. Ray HA, Trope M. Periapical status of endodontically treated teeth in relation to the technical quality of the root filling and the coronal restoration. Int Endod J 1995;28(1):12-8.

3. Naumann N, Kiessling S, Seemann R. Treatment concepts for restoration of endodontically treated teeth: A nationwide survey of dentists in Germany. J Prosthet Dent 2006;96:332-8.
4. Heydecke G, Butz F, Strub JR. Fracture strength and survival of endodontically treated maxillary incisors with approximal cavities after restoration with different post and core systems: an in-vitro study. J Dent 2001;29:427-33.

5. Pontius O, Hutter JW. Survival rate and fracture strength of incisors restored with different post and core systems and endodontically treated incisors without coronoradicular reinforcement. J Endod 2002;28:710-5.

6. Balkenhol M, Wostmann B, Rein C, Ferger P. Survival time of cast post and cores: a 10-year retrospective study. J Dent 2007;35:50-8.

7. Cheung W. A review of the management of endodontically treated teeth: post, core and the final restoration. $J$ Am Dent Assoc 2005;136(5):611-9.

How to cite: Bansal K, Bansal BP, Aggarwal N, Nischal M. Retreatment of permanent incisors with fibre and cast metalposts. Indian J Conserv Endod 2020;5(1):37-9. 